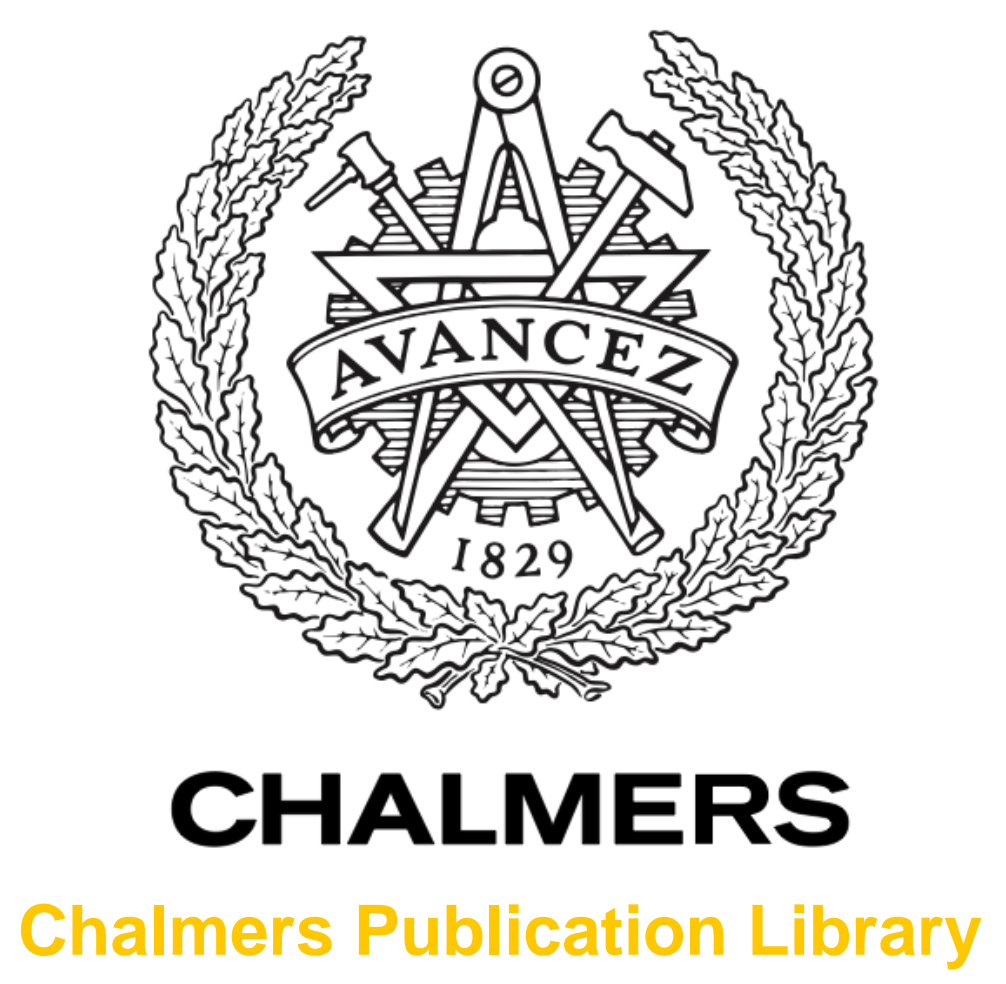

\title{
Diffraction and Pattern Perturbation Effects in Offset Gregorian Reflector Antennas with Wideband Feeds
}

This document has been downloaded from Chalmers Publication Library (CPL). It is the author's version of a work that was accepted for publication in:

\section{IEEE International Symposium on Antennas and Propagation \& USNC/URSI National Radio Science Meeting}

Citation for the published paper:

Villiers, D. ; Ivashina, M. (2015) "Diffraction and Pattern Perturbation Effects in Offset Gregorian Reflector Antennas with Wideband Feeds". 2015 IEEE International Symposium on Antennas and Propagation \& USNC/URSI National Radio Science Meeting pp. 15201521.

http://dx.doi.org/10.1109/APS.2015.7305149

Downloaded from: http://publications.lib.chalmers.se/publication/227916

Notice: Changes introduced as a result of publishing processes such as copy-editing and formatting may not be reflected in this document. For a definitive version of this work, please refer to the published source. Please note that access to the published version might require a subscription. 


\section{Diffraction and Pattern Perturbation Effects in Offset Gregorian Reflector Antennas with Wideband Feeds}

\author{
Dirk I. L. de Villiers \\ Department of Electrical and Electronic Engineering, \\ Stellenbosch University, Stellenbosch, South Africa, \\ ddv@sun.ac.za
}

\author{
Marianna V. Ivashina \\ Antenna Group, Department of Systems and Signals, \\ Chalmers University of Technology, Gothenburg, Sweden, \\ marianna.ivashina@chalmers.se
}

\begin{abstract}
The offset Gregorian reflector system of the Square Kilometer Array radio telescope is required to operate with wideband feed (WBF) antennas down to very low frequencies, where reflectors become (electrically) relatively small. The considered WBF technologies are the log-periodic (Eleven) antenna and the quadruple-ridged flared horn. This paper investigates some of the performance degradation effects that are specific for the low frequencies and typical for these WBFs, including the feed pattern perturbations and diffraction. Several performance metrics, such as the antenna receiving sensitivity and side lobe levels are considered.
\end{abstract}

Index Terms - Radio astronomy reflector antennas, wideband feeds.

\section{INTRODUCTION}

Large reflector antenna systems, such as Gregorian and Cassegrain antennas, are traditionally designed to operate at high frequencies, with single or multiple narrowband feeds. However, with a rapid development towards the multiantenna systems, i.e. e.g. systems consisting of several smaller reflector antennas fed with wideband feeds (WBFs), high performance can be achieved over much larger frequency bands. One example of such advanced systems is the offset Gregorian reflector antenna of the future Square Kilometer Array (SKA) radio telescope [1]. This system is required to operate with WBFs down to very low frequencies, where the reflectors are (electrically) relatively small. Although the performance of the considered antenna feeds - the Eleven antenna and the Quadruple-Ridged Flared Horn -, as predicted through the high-frequency asymptotic techniques, is known [2], the goal of this paper is to put in perspective the different contributions to the reduced performance we observe when moving to the lower frequencies. We will demonstrate that some of these are related to the small size of the dishes (diffraction effects), while the others are due to the unique properties of the WBFs (feed pattern perturbation effects). The results of this analysis can provide an important input to the SKA community for improving the current baseline designs.

\section{WIDEBAND FEED PATTERN DESCRIPTION}

The feeds of interest here have been optimized for use in the $350 \mathrm{MHz}-1050 \mathrm{MHz}$ band (Band 1) of the SKA, with initial results presented in [2]. The results in [2] reveal broadly similar performance of the two feeds when used in the SKA optics, with the Eleven feed displaying more frequency variation and the QRFH higher cross-polarization levels. When designing reflector systems, it is desirable to have feeds with as little as possible variation in frequency of the radiation patterns. A study in [3] describes the effect on axi-symmetrical reflector performance of some of the main perturbations in the feed radiation patterns, namely variations of the feed illumination taper and its pattern symmetry. Plots, illustrating these quantities for both feeds, are shown in Fig. 1, where the feed taper is calculated as an average over azimuthal cuts at $53^{\circ}$ polar angle, and the pattern symmetry is defined as the absolute difference between the E- and H-plane pattern edge tapers.

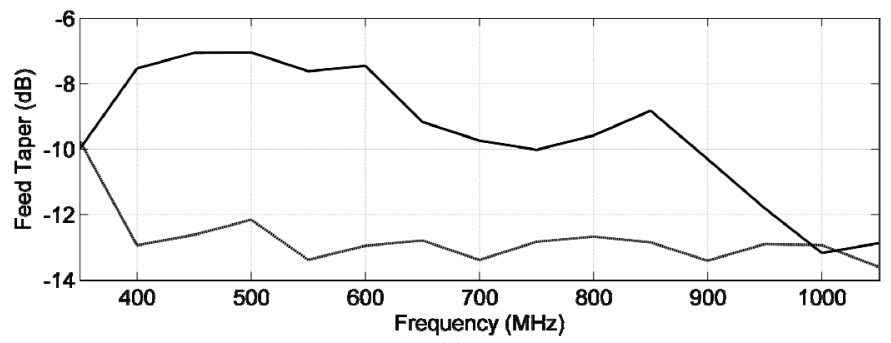

(a)

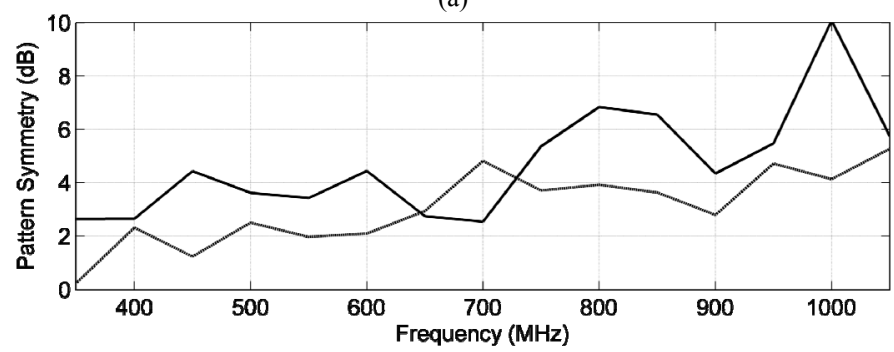

(b)

Fig. 1. Feed taper (a) and pattern symmetry (b) of the Eleven feed (solid line) and the QRFH feed (dashed line).

\section{LOW FREQUENCY DISH EFFECTS}

As the electrical size of reflector system is reduced, diffraction from the sub- and main reflector edges alters the aperture field distribution, and thus secondary pattern performance, from that expected if the system is designed or analyzed in a geometric optics sense. The effects of diffraction include decreased aperture efficiency [4], increased crosspolarization levels [5], as well as increased side lobe level (SLL). An ideal Gaussian feed pattern, with $12 \mathrm{~dB}$ edge taper, was analyzed (using GRASP [6]) on the offset Gregorian 
system described in [1] with an extended sub-reflector of $5 \mathrm{~m}$ chord length and a $53^{\circ}$ subtended angle over the Band 1 frequency range, and the symmetry plane cuts of the normalized directivity secondary patterns are shown in Fig. 2 for several frequencies.

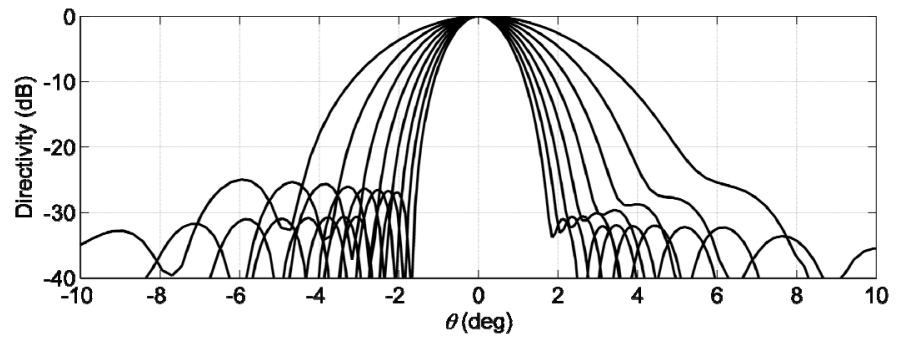

Fig. 2. Normalized secondary pattern directivity in the symmetry plane of an offset Gregorian reflector. A $12 \mathrm{~dB}$ edge taper Gaussian feed is used, and plots are shown for the frequency range [350:100:1050] $\mathrm{MHz}$.

Note how the patterns become more asymmetrical for the lower frequencies (wider patterns), with a coma lobe which merges into a shoulder appearing on the side opposite from the sub-reflector (positive $\theta$ in this case) - effectively increasing the first SLL with decreased frequency. This effect is purely due to asymmetrical diffraction from the dishes, since the feed is perfectly symmetric in all cases.

\section{FeEd Perturbation EfFects}

The two main feed pattern perturbations discussed in Section II was evaluated by simulating ideal Gaussian feeds on the same reflector system as in Section III. First the effect of varying feed taper and frequency on SLL is illustrated in Fig. 3 (the effect on sensitivity for similar cases has been discussed previously in [7]). The expected increase in SLL with smaller feed tapers is obvious, as well as the increase, in especially the first SLL, with decreasing frequency - as expected from Fig. 2. The cross-polarization levels are found to not be a strong function of feed taper, but the strong frequency dependence described in [5] is again evident.

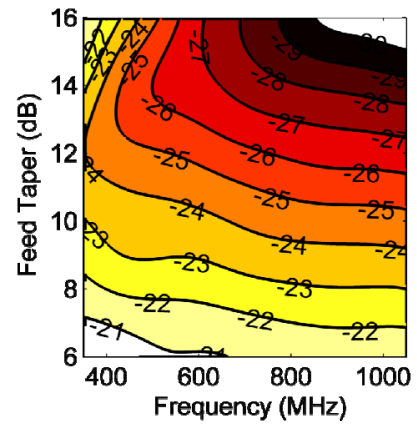

(a)

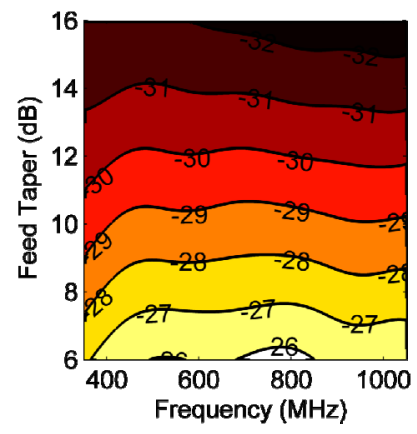

(b)
Fig. 3. First (a) and second (b) SLL (in dB) of an ideal Gaussian feed with varying edge taper, illuminating an offset Gregorian reflector, over the Band 1 frequency range.

A similar set of simulations was performed to evaluate the effects of pattern symmetry. Here the feed taper was kept constant in one principle plane at $12 \mathrm{~dB}$, while the pattern in the other plane was varied. Variation in both planes and both linear polarizations were investigated, and the case displaying the largest variation is shown in Fig. 4. Here the polarization is in the symmetry plane of the system, with the E-plane pattern varied (results are similar for the orthogonal polarization with H-plane variation). Main points to note from the results are the expected increase in SLL for wider patterns (negative values of pattern symmetry), the sensitivity of crosspolarization level to asymmetric patterns (as in [3]), and then relative insensitivity of the sensitivity (shown here in the $30^{\circ}$ feed down tipping direction) to pattern asymmetry. The sensitivity to reduced electrical size is obvious in all cases though, and this remains the main limiting factor in the Band 1 SKA design.

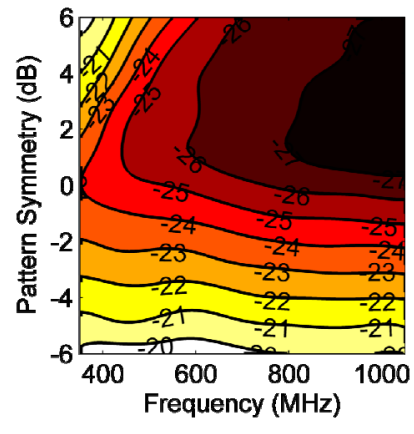

(a)

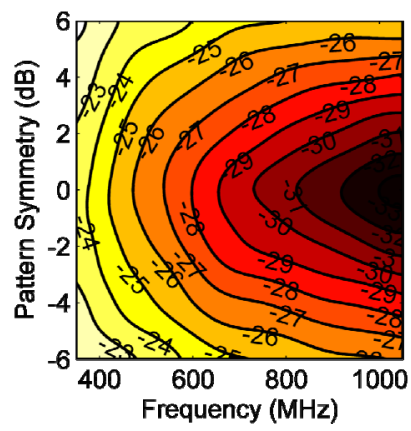

(c)

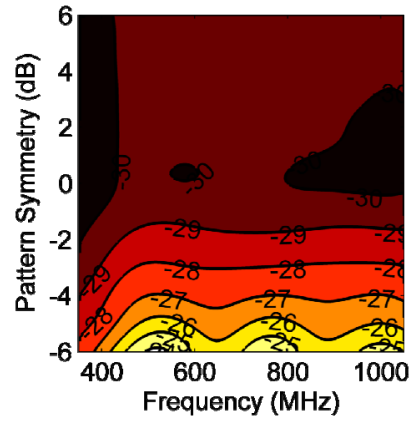

(b)

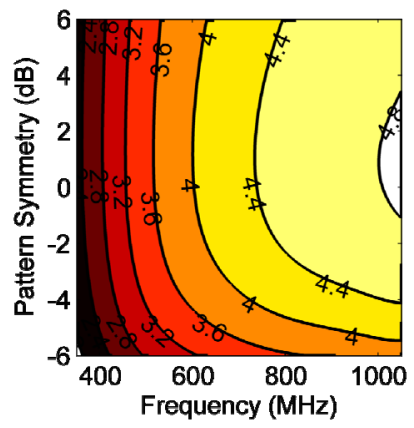

(d)
Fig. 4. First (a) and second (b) SLL, as well as Jones cross polar level (c), within the $3 \mathrm{~dB}$ main beam region (in $\mathrm{dB}$ ), and the sensitivity (d) $\left(\right.$ in $\mathrm{m}^{2} / \mathrm{K}$ ) of an ideal Gaussian feed with varying pattern symmetry, over the Band 1 frequency range.

\section{REFERENCES}

[1] I. P. Theron, R. Lehmensiek, and D. I. L. de Villiers, "Towards an optics design for SKA," IEEE AFRICON, Mauritius, pp. 1313-1317, Sep. 2013.

[2] M. V. Ivashina, C. Bencivenni, O. Iupikov, and J. Yang, "Optimization of the 0.35-1.05 GHz Quad-Ridged Flared Horn and Eleven Feeds for the Square Kilometer Array Baseline Design", Int. Conf. Electromagnetics. Adv. Appl., Palm Beach, Aruba, pp. 513 - 516, Aug., 2014.

[3] M. Safak, "Forward Radiation from Axially Symmetric Reflector Antennas," IEEE Trans. Antennas Propag., vol. AP-32, no. 9, pp. 893901, Sept. 1984.

[4] D. I .L. de Villiers, “Offset Dual Reflector Antenna System Efficiency Predictions Including Sub-Reflector Diffraction", IEEE Antennas Wireless Propag. Lett., vol. 10, 2011, pp. 947 - 950.

[5] T. S. Bird, "Investigation of crosspolarisation in offset Cassegrain antennas", Electr. Lett., vol. 17, no. 17, pp. 585-586, Aug. 1981.

[6] TICRA, GRASP10, Version 10.3.0, Copenhagen, Denmark. [Online].Available: http://www.ticra.com

[7] D. I. L. de Villiers and R. Lehmensiek, 'Sensitivity Performance of the SKA Offset Gregorian Reflector candidates with Ideal Feeds', IEEE Int. Symp. Antennas and Propag, Memphis, USA, pp. 1477-1478, July, 2014. 